\title{
The LHCb Outer Tracker Detector Design and Production
}

\author{
L.B.A. Hommels \\ On behalf of the LHCb Outer Tracker group \\ NIKHEF Office N341 \\ P.O. Box 41882 \\ 1009 DB Amsterdam \\ the Netherlands \\ email: hommels@nikhef.nl
}

\begin{abstract}
The Outer Tracker detector of the LHCb experiment is a gas filled detector based on straw tube technology. It comprises three stations each consisting of multiple layers of wires with both vertical orientation and $\pm 5^{\circ}$ stereo angles. The stations are designed to be built of modules which are mechanically stable, gas tight and capable of stand-alone operation. This paper describes the design and mass production procedures of Outer Tracker modules and discusses the quality assurance programme. Test results on the first modules produced are included.
\end{abstract}

\section{INTRODUCTION: THE LHCB EXPERIMENT}

$\mathrm{LHCb}$ is an experiment at the LHC dedicated to study $\mathrm{CP}$ violation in $\mathrm{B}$ decays with high precision [1]. The production of $\mathrm{b} \bar{b}$ pairs at the LHC will be peaked in the forward direction, therefore $\mathrm{LHCb}$ is designed as a single-arm spectrometer covering 10-300 mrad in polar angle. Fig. 1 depicts the detector in its cavern. The $\mathrm{LHCb}$ experiment aims at having flexible, efficient and precise $b \bar{b}$ reconstruction using the tracking system consisting of a vertex locator and main tracker, the RICH1 and RICH2 particle identification detectors, a muon system and electromagnetic and hadronic calorimeters.

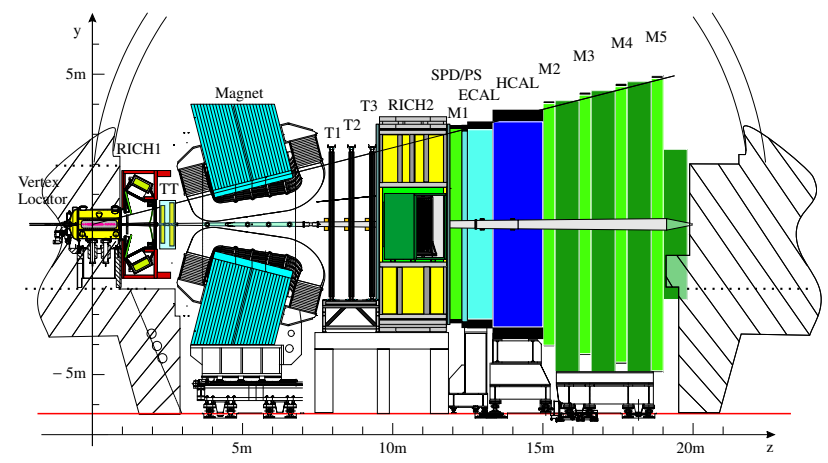

Fig. 1. Side view of the $\mathrm{LHCb}$ detector in its cavern. The stations of the main tracker are indicated with $\mathrm{T} 1$ to $\mathrm{T} 3$.

\section{The LHCB OUter TRACKeR}

\section{A. LHCb main tracker performance}

The main tracking volume in $\mathrm{LHCb}$ comprises 3 tracker stations installed over $3 \mathrm{~m}$ length in the field-free region behind the dipole magnet. In front of the magnet a tracking station is installed mainly for triggering purposes.

The distribution of particle fluxes in LHCb is very high near the beam pipe but falls off rapidly with increasing distance from the beam axis. To limit detector occupancy, the main tracking detector is split in an Inner Tracker consisting of silicon microstrips with fine granularity around the beampipe and an Outer Tracker (OT) covering the greater part of the tracking volume which is a drift-chamber detector using strawtube technology. A view of the three main tracker stations is depicted in Fig.2.

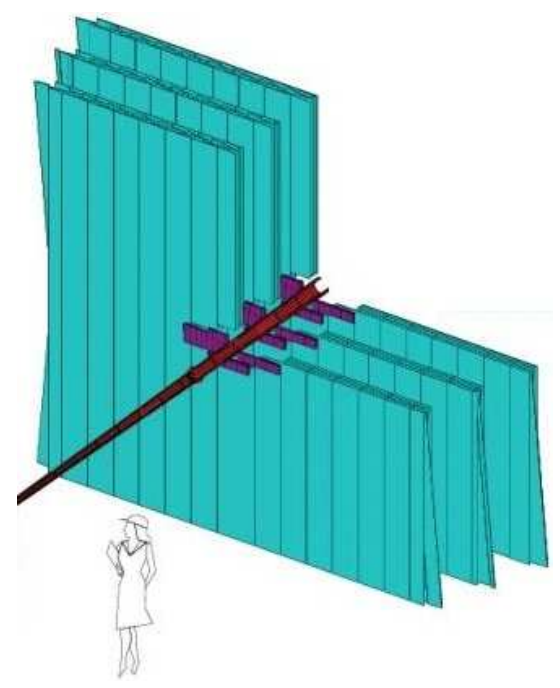

Fig. 2. The three main tracker stations with their Inner and Outer Tracker detectors and the LHCb beampipe.

The tracking stations are optimized for momentum mea- 


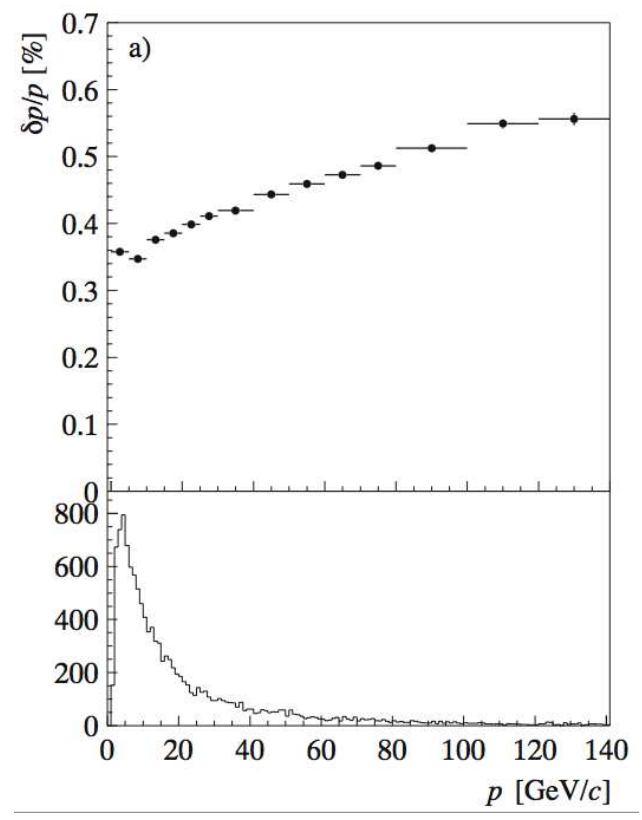

Fig. 3. Momentum resolution on reconstructed tracks as a function of track momentum. For comparison, the momentum spectrum of B-decay particles is shown in the lower part of the plot.

surement: the stations have good spatial resolution in the bending plane and sufficient pattern-recognition capabilities in the non-bending plane of the magnet. Each outer tracker station consists of multiple layers of wires with both vertical orientation and $\pm 5^{\circ}$ stereo angles.

Simulations with the current detector layout show a good average momentum resolution of $\delta p / p<0.6 \%$ over a wide momentum range $5<p<140 \mathrm{GeV}$ as can be seen from the plot in Fig.3. Multiple scattering contributes to the resolution with approximately $200 \mu \mathrm{m}$, determining the minimum resolution requirement of the OT.

\section{B. Outer Tracker characteristics}

The Outer Tracker has to operate in the harsh radiation environment of the $\mathrm{LHCb}$ experiment. Therefore the detector has to be built from materials that are radiation hard. The ageing phenomena of straw tubes and detector gases has been studied extensively [2] to ensure reliable detector operation for its intended lifetime of 10 years.

Detectors used in an LHC experiment need to be fast given that the time between consecutive bunch crossings is only 25 ns. When accepting a drift-time window of 3 bunch crossings at maximum, the speed demands can be met by using straw tubes with an inner diameter of $5 \mathrm{~mm}$ with an $\mathrm{Ar}-\mathrm{CO}_{2}$ mixture as drift gas.

A pre-amplifier with short input peaking time and low noise is required for accurate timing of the leading edges of the straw tube signals. The ASDBLR, developed by the ATLAS TRT collaboration in radiation hard DMILL technology [4] matches the requirements. It features active baseline restoration to suppress the slow ion-tail signals and hence its deadtime is reduced to a few tens of ns.
The ASDBLR output signals are read out by the OTIS, a custom TDC developed for the OT using radiation hard technology [5]. It digitizes and stores ASDBLR pulses during the L0 trigger latency of $4 \mu \mathrm{s}$ and contains a derandomizer buffer to cover trigger rate fluctuations. The OTIS output data is serialized and optical links are used for shipping the data to the off-detector DAQ system [3].

\section{OUter TRACKER DESIGN}

\section{A. Station Design}

The design of the three Outer Tracker stations is modular. Stations are built from separate modules suspended in an aluminium frame, as shown in Fig.4. To define the postion of the modules relative to the frame, the frame is equipped with precision dowel pins to mount the modules. The necessary cabling and services are incorporated in the frame design.
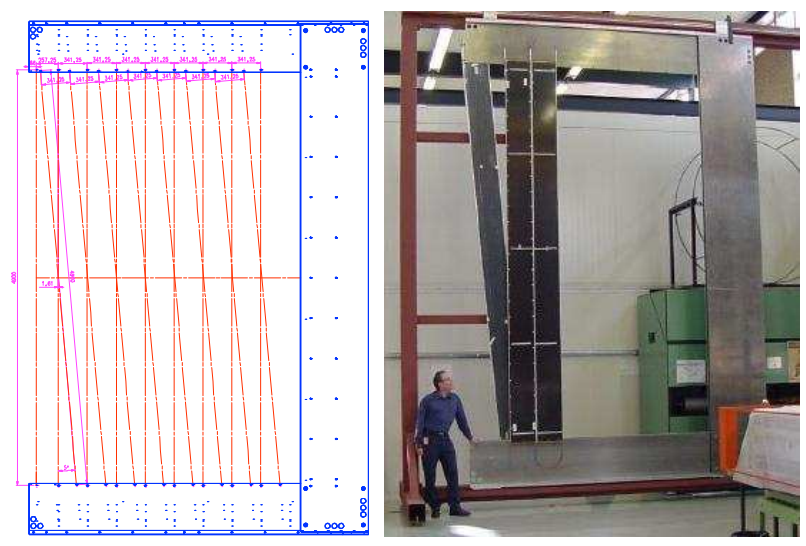

Fig. 4. Design of a station frame on which the modules are suspended. The photo on the right shows a test frame with several modules from the first production batch mounted onto the frame.

The greater part of the modules stretches along the full height of the stations. Covering the area around the Inner Tracker requires modules of different sizes as can be seen in Fig.5.

\section{B. Module Design}

Each module is mechanically stable, gas tight and capable of stand-alone operation. One module contains up to 256 straw tubes arranged in two staggered monolayers. The module cross-section is depicted in Fig.6.

A module consists of two panels and two sidewalls to form a mechanically stable and gas-tight box. Straw tubes are glued to a panel using a high-precision jig to accurately define the straw tube position and straightness. The panels are made of a Rohacell core covered with carbon skins. In addition, the inner side has an extra layer of a polyimide (Kapton)-aluminium laminate for gas tightness and electrical shielding. Carbonfibre sidewall strips connect the module halves and provide additional stiffness to the module.

The panels and sidewalls provide a secondary gas volume surrounding the straw tubes. To avoid pollution of the counting 
gas with contaminating elements entering through diffusion or small leaks, the secondary volume is flushed independently from the primary volume defined by the straw tube walls.

\section{Straw Tube Technology}

In the modules, straw tubes are electrically separated at the equatorial plane for occupancy reasons, thereby limiting the maximum straw tube length to $2.5 \mathrm{~m}$. Every $80 \mathrm{~cm}$, wire locators support and centre the wire inside the straw tubes. At the straw tube ends, endpieces are inserted to keep the wire centered and ensure a proper flow of the counting gas into the straw tubes. A picture of the aforementioned pieces is included in Fig.7.

A straw tube is wound from two layers of foil material as can be seen in Fig.7. An inner layer of carbon-doped Kapton (Kapton XC) acts as a cathode for the collection of the positive ions. The outer layer, made of a Kapton XCaluminium laminate provides shielding and limits the crosstalk amongst neighbouring channels. Together with the anode wire the aluminium winding forms a transmission line for effective transport of the fast response signals.

\section{Front-End Electronics Interface}

Feed-Through boards are incorporated at both module ends. These provide the electrical connection between the signal wires (anodes) inside the gas box and the Front-End Electronics outside. The board also provides the connection to a common ground reference via a continuous ground layer following the path of the signal.

To provide a reliable and lasting ground contact, the cathode of the straw tubes is extended and soldered onto the FeedThrough board. Also the (inner) Aluminum side of the panels are soldered onto the GND layer of the board. A special contact spring provides then the ground connection to the Front-End electronics boards, as can be seen in Fig.8. The

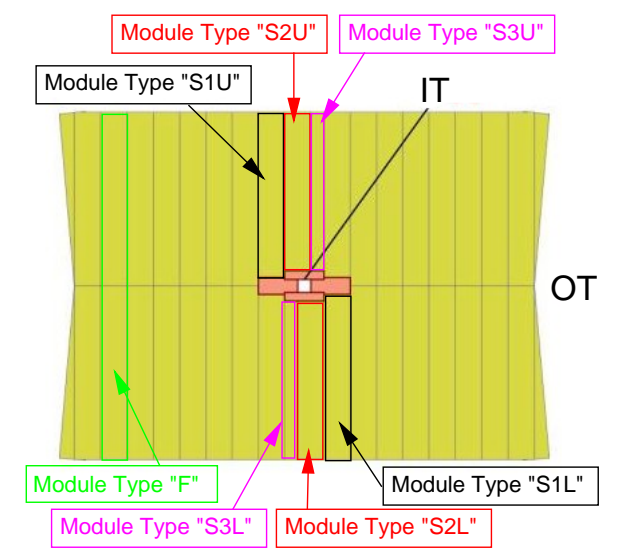

Fig. 5. A Tracker station with the various sizes of Outer Tracker modules indicated. design of the matching Front-End electronics boards is advanced, Fig. 9 contains photographs of dedicated pre-amplifier and TDC prototype boards.

\section{Outer Tracker Module Production}

Mass production of OT modules has recently started after an extensive R\&D programme has been completed including beam-tests on prototypes.

\section{A. Module assembly}

The modules are initially assembled in module halves. The assembly of a half-module comprises the following steps:

1) Straw tubes are cut to length and equipped with wirelocators and endpieces.

2) The straw tubes are placed in a high-precision jig together with the Feed-Through PCB's. The straw ground contacts are soldered to the PCB (see Fig.8).

3) The module panel is positioned in a dedicated tool and glued to the straws-PCB ensemble.

4) After the glue has cured, anode wires are strung. Tests are performed on individual channels; eventual bad wires are replaced.

At this stage, module internals are still reachable. If two module halves have successfully passed a series of tests described in the section below, they are joined. A photo of the assembly setup is included in Fig.10. After a check on gas tightness, correct operation of the module is ensured through a series of tests which are discussed in the following section.

\section{B. Module tests and results}

Before two module halves are joined together (see Fig.10), module internals are still reachable. Individual channels are subjected to the tests described below, with the opportunity for repairs in the case of failures [6].

- At the ends of the straw tube, the wire position is measured. The deviation should be within $100 \mu \mathrm{m}$ from the straw tube centre.

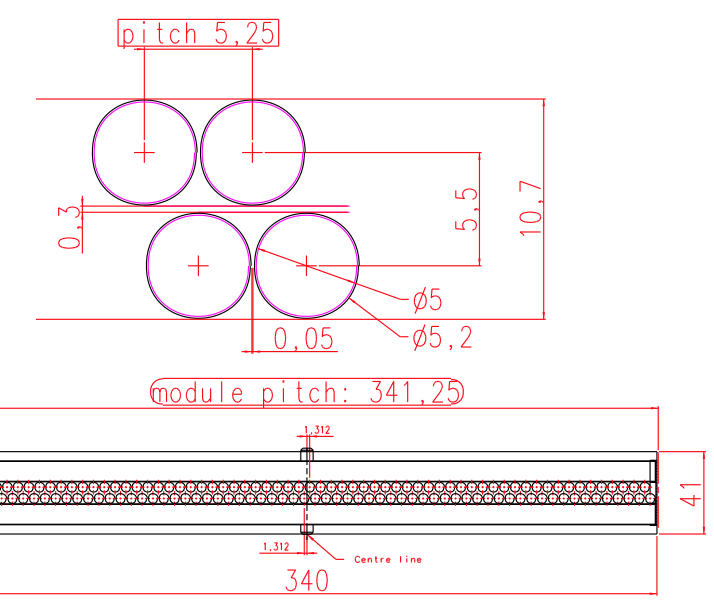

Fig. 6. Cross-section of a module. The top drawing depicts the arrangement of straws inside the module box. 


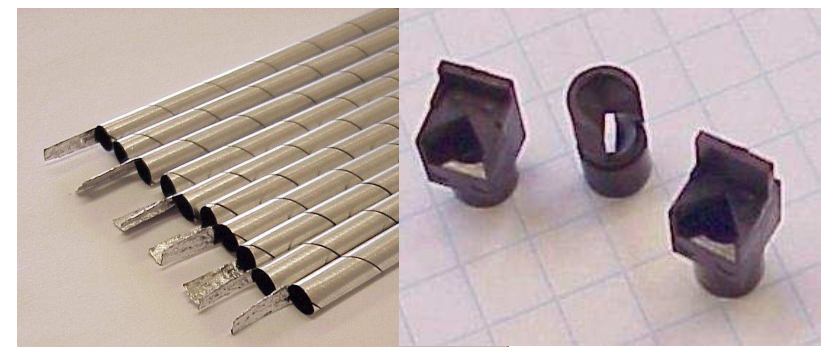

Fig. 7. On the left: straw tubes with extended cathode contacts. On the right photo a wirelocator and two endpieces can be seen.

- After the wires are strung and soldered, the wire tension is determined by detecting the resonance frequency in a magnetic field. When a deviation from the nominal tension is detected, the wire is replaced.

- When the wire tension of all wires are checked to be within the specifications, they are put on high voltage (HV) while in air. During the ramp-up of the voltage the wire current is monitored continuously. Wires with abnormal current values are replaced.

Once all channels on the module halves have passed these tests, the halves are joined to a module. After a check on gas-tightness, modules are subject to so-called HV-training. Initially the module is flushed with counting gas after which $\mathrm{HV}$ is applied. It is observed that wire currents are initially high but stabilize at normal values in a few hours. The plots in Fig. 11 show this effect for a few typical wires of a module.

\section{Quality Assurance}

For validation of the production process, the modules produced so far are subjected to a test using a radioactive source. A ${ }^{90} \mathrm{Sr} \beta^{-}$line source emitting electrons up to about $2.3 \mathrm{MeV}$ is moved along the sense wires in $1 \mathrm{~cm}$ steps. For every

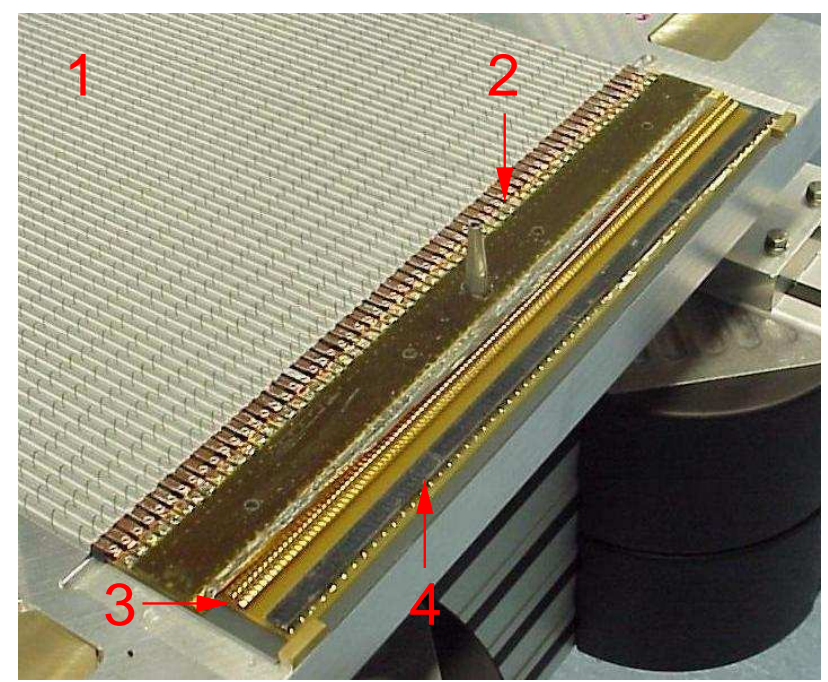

Fig. 8. A half module assembly including the Feed-Through board. "1" indicates the straw tubes with their grounding contacts marked at " 2 ". At " 3 " the spring loaded ground contact can be seen and " 4 " marks the connector to the Front-End electronics.

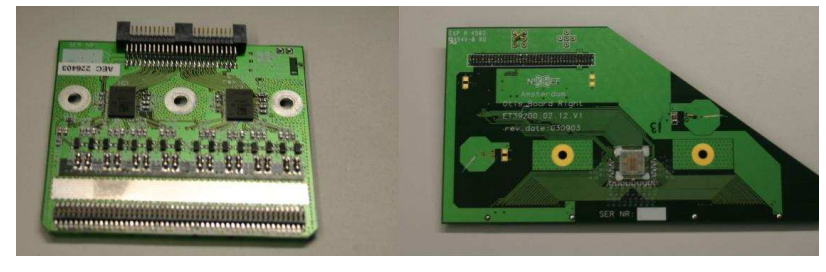

Fig. 9. Photographs of a pre-amplifier board with two ASDBLR chips (left) and a OTIS TDC board (right).

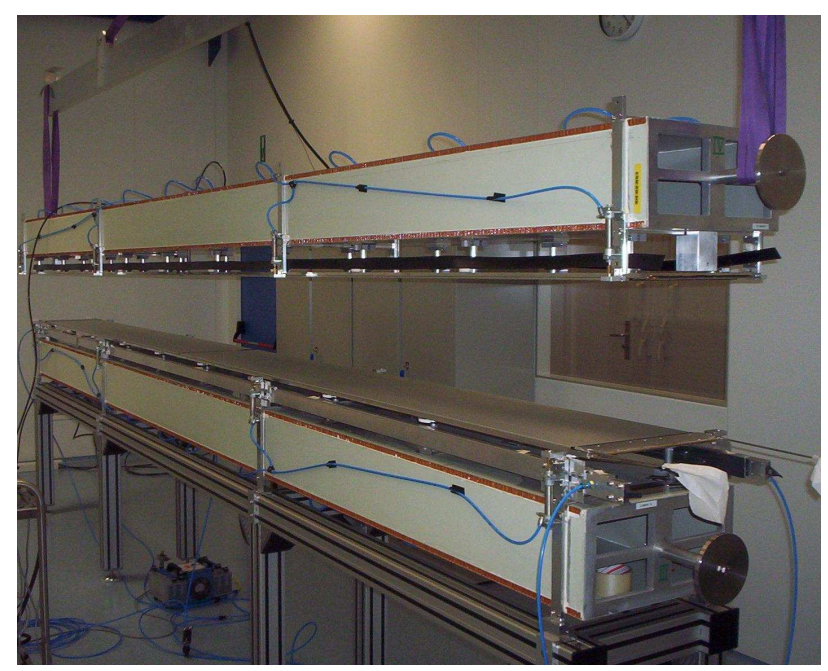

Fig. 10. Photograph of the module assembly tool. Both the upper and lower support structures hold a half module which are to be joined shortly.

step the wire currents are measured. A picture of the setup is included in Fig.12. The inset shows a measured plot of the source intensity profile, for which the module response is corrected.

Among other effects, the deviation of the sense wire with respect to the straw tube centre contributes to non-uniformities in the current response to the illumination by the source.

In Fig.13, the complete result of a ${ }^{90} \mathrm{Sr}$ scan is plotted. All hidden structures of the module are clearly visible. The vertical white lines show the drop in the current at the position of the wire locators and the straw separation zone in the middle of the module. With such a scan, displaced wirelocators could be spotted immediately. Also, wires showing abnormally high or low currents can be marked as problematic.
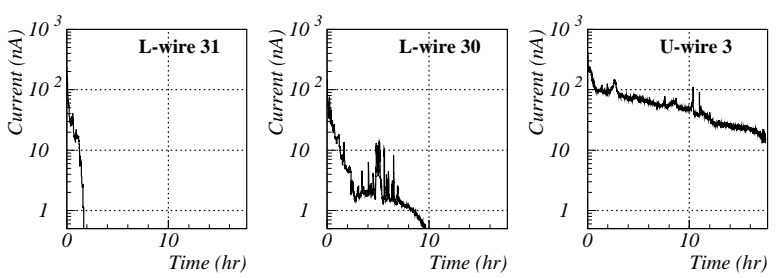

Fig. 11. For a few typical wires the development in time of the channel dark current while on high voltage is plotted. 


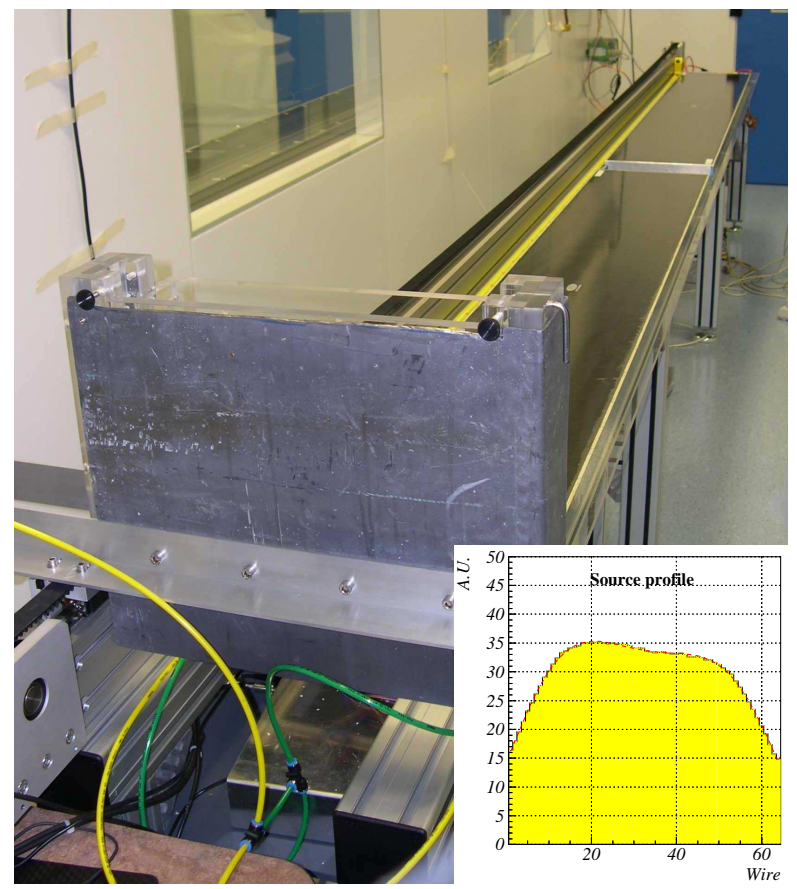

Fig. 12. Photograph of the ${ }^{90} \mathrm{Sr}$ scanner tool. A drive belt (on the left) displaces the shielded source over the module on the table below. The inset shows the intensity profile of the ${ }^{90} \mathrm{Sr}$ source.

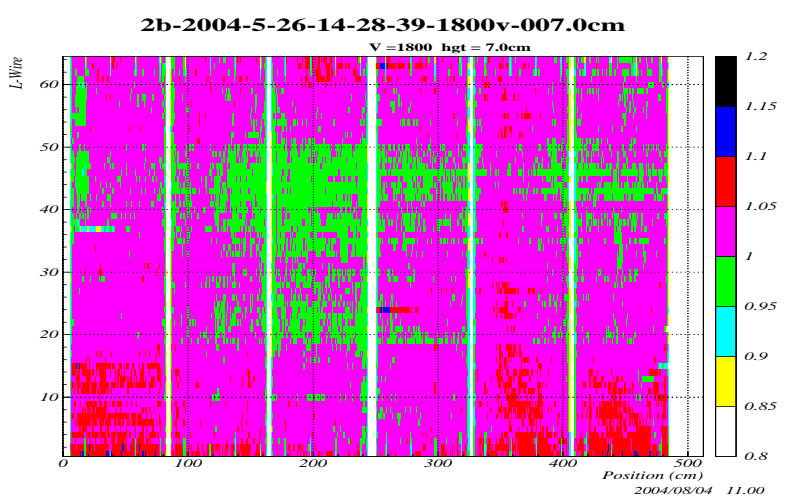

Fig. 13. Result of a ${ }^{90} \mathrm{Sr}$ scan of a module. Plotted are the relative wire currents, corrected for the source profile.

The ${ }^{90} \mathrm{Sr}$ scanning setup has proven itself as an essential tool to provide feedback on possible improvements of the module assembly procedures.

\section{CONCLUSION}

The mass production of $\mathrm{LHCb}$ Outer Tracker modules is under way. The current production method has lead to stable and gas-tight modules without wire failures. The checks and tests described earlier indicate excellent channel uniformity and no irregularities along the wire lengths.

\section{REFERENCES}

[1] LHCb Collaboration, Technical Design Report 6, 2001-24, Geneva: September 2001.
[2] S.Bachmann et al. Ageing studies for the straw tube detectors of the LHCb Outer Tracking system, Contribution to this conference.

[3] U.Uwer et al. Front-End Electronics of the LHCb Outer Tracker, Contribution to this conference.

[4] Bevensee, B., Newcomer, M., Van Berg, R., Williams, H. H., Progress Report on the Development of a Bipolar ASIC for the ATLAS Transition Radiation Detector, ATLAS internal note INDET-NO-080, Geneva: September 1994.

[5] http://wwwasic.kip.uni-heidelberg.de/Projects/lhcbotis.html

[6] van Apeldoorn, G. et al., Outer Tracker Module Production at NIKHEF - Quality Assurance, CERN-LHCb-2004-078, Geneva : CERN, October 2004. 